\title{
VIEWPOINT
}

\section{Crisis Resource Management and High-Performing Teams in Hyperacute Stroke Care}

\author{
Phavalan Rajendram ${ }^{1 \dagger}$, Lowyl Notario ${ }^{2 \dagger}$, Cliff Reid ${ }^{3 \dagger}$, Charles R. Wira ${ }^{4 \dagger}$, Jose I. Suarez ${ }^{5 \dagger}$, Scott D. Weingart ${ }^{6 \dagger}$ \\ and Houman Khosravani ${ }^{*}$ (D)
}

(C) 2020 Springer Science+Business Media, LLC, part of Springer Nature and Neurocritical Care Society

\begin{abstract}
Background and Purpose: Management of stroke patients in the acute setting is a high-stakes task with several challenges including the need for rapid assessment and treatment, maintenance of high-performing team dynamics, management of cognitive load affecting providers, and factors impacting team communication. Crisis resource management (CRM) provides a framework to tackle these challenges and is well established in other resuscitative disciplines. The current Coronavirus Disease 2019 (COVID-19) pandemic has exposed a potential quality gap in emergency preparedness and the ability to adapt to emergency scenarios in real time.
\end{abstract}

Methods: Available resources in the literature in other disciplines and expert consensus were used to identify key elements of CRM as they apply to acute stroke management.

Results: We outline essential ingredients of CRM as a means to mitigate nontechnical challenges providers face during acute stroke care. These strategies include situational awareness, triage and prioritization, mitigation of cognitive load, team member role clarity, communication, and debriefing. Incorporation of CRM along with simulation is an established tool in other resuscitative disciplines and can be incorporated into acute stroke care.

Conclusions: As stroke care processes evolve during these trying times, the importance of consistent, safe, and efficacious care facilitated by CRM principles offers a unique avenue to alleviate human factors and support highperforming teams.

Keywords: Stroke, Critical care, Pandemics, Patient care team

\section{Introduction}

Assessment of patients with stroke for hyperacute treatments such as thrombolysis and thrombectomy must be timely, precise, and effective to achieve the best clinical

\footnotetext{
*Correspondence: houman@stroke.dev

†Phavalan Rajendram and Lowyl Notario Co-first authors. Cliff Reid,

Charles R. Wira, Jose I. Suarez and Scott D. Weingart have been contributed equally to this work.

1 Brain Resuscitation Lab, Neurology Quality and Innovation Laboratory (NQIL), Division of Neurology, Department of Medicine, Sunnybrook Health Sciences Centre, University of Toronto, Room H335 - 2075 Bayview Avenue, Toronto, ON M4N 3M5, Canada

Full list of author information is available at the end of the article
}

outcomes. Stroke care has advanced significantly over the years with the emergence of new hyperacute therapies including late-window thrombectomy, advanced imaging, and the creation of systems of care. The current Coronavirus Disease 2019 (COVID-19) pandemic has exerted stress on existing stroke clinical pathways, exposing a potential quality gap in terms of emergency preparedness. This is especially poignant during the hyperacute phase as providers form ad hoc teams to evaluate and resuscitate patients.

Elements at play include patient factors, health-care resources, team dynamics, and well-being of providers. These notions span the chain of survival in stroke from

\section{实 Springer}


hyperacute assessment, to admission, and discharge. Specific to hyperacute assessment during the COVID-19 pandemic, the concept of a protected code stroke (PCS) has emerged, highlighting the importance of screening, adherence to infection prevention and control measures, and coordinated team responses [1]. Nonetheless, highperforming teams support good outcomes not only at the resuscitation phase, but apply to all subsequent care including neurocritical care, inpatient stroke unit, and discharge planning. Central to a high-performing team are principles of CRM and their timely application to stroke care.

Knowledge and mastery of technical skills are key to the successful execution of a code stroke. However, there are a myriad of nontechnical skills. CRM provides a framework for these skills including situational awareness, triage and prioritization, cognitive load reduction, role clarity, communication, and debriefing [2]. These principles were originally described as "crew resource management" in the aviation industry, where human error can similarly have disastrous consequences. CRM is increasingly recognized within medicine and has already taken root in specialties such as emergency medicine [3], critical care medicine [4], and anesthesia [5]. These specialties share a common thread with hyperacute stroke care. The unifying premise is resuscitation that bridges stroke with neurocritical care.

Providers in code strokes face a high-acuity scenario and effectively function as "brain resuscitationists." The COVID-19 pandemic further accentuates the need for routine CRM to ensure an effective resuscitation when there are additional health-care system pressures. Present day offers a unique epoch to introduce CRM within stroke care so that in the event of the next crisis, stroke systems and providers are well equipped to deal with adversity. Herein, we outline key aspects to consider in implementing CRM principles in hyperacute stroke care during the COVID-19 pandemic and beyond.

\section{Overarching Principles}

CRM combines several essential elements that span the team, environment, and individual provider. In the resuscitation setting, it has been proposed that these nontechnical components be considered prior to hands-on patient assessment as a "zero point survey," prior to the primary survey and resuscitation (Fig. 1) [6]. Implementing CRM principles in day-to-day practice can enhance team cohesion and satisfaction, thereby enhancing team performance. This can potentially translate to several improved outcomes including a culture of safety, post-resuscitation length of stay, patient satisfaction, and survival [2]. Utilization of simulation-based learning reinforces CRM principles providing cumulative marginal gains in operational performance. CRM principles in conjunction with stakeholder engagement should be used to develop pre-defined checklists for the initial evaluation, management, and debriefing phases of resuscitation based on local practice parameters.

\section{Situational Awareness}

The term situational awareness is one of the prime directives of resuscitation. It is well utilized and established as part of other resuscitation protocols. The term encompasses full awareness of self, team, and environment.

To be effective at situational awareness, one needs to recognize "cues" in the environment that are pertinent to assessment of the patient, comprehension of the environment, and projection of future status. These cues then require synthesis to arrive at a list of focused differential diagnoses and patient trajectory. For effective synthesis, providers must also be aware of cognitive and diagnostic biases. Maintenance of situational awareness can be compromised by factors including over-stimulation, lack of clear loops of communication, lack of role designation, and accelerated clinical deterioration. This element of maintenance of awareness requires deliberate practice (e.g., simulation training).

The importance of situational awareness is best illustrated in code strokes where a patient's hemodynamic and/or airway status is tenuous. Providers must perceive all available clues, predict impending decompensation, and initiate appropriate resuscitative measures. Synthesis and continual re-evaluation of cues leads to a projection of near-term deterioration, thus avoiding decompensation in or en route to the scanner or neuroangiography suite. Situational awareness is important for all team members involved, as it can be easy for a member to become focused on their assigned task and lose sight of the larger clinical picture. Having set checkpoints where the team leader can summarize findings and establish priorities can enhance situational awareness for the team. "There is no emergency in a pandemic" [7]. For a PCS, situational awareness ensures safety of the team and minimizes contamination of the environment. Placement of a mask on the patient may be a task that is forgotten, which can be mitigated by situational awareness. Similarly, it is easy to overlook the designation of a safety leader, increasing the risk of poor adherence to meticulous donning and doffing of personal protective equipment.

\section{Triage and Prioritization}

During resuscitation, the number of tasks can be overwhelming and often not congruent with immediately available resources. Prioritization of medical tasks (e.g., neurologic exam, control of blood pressure) and nontechnical tasks (e.g., communication with the other 


\section{Zero Point Suney}

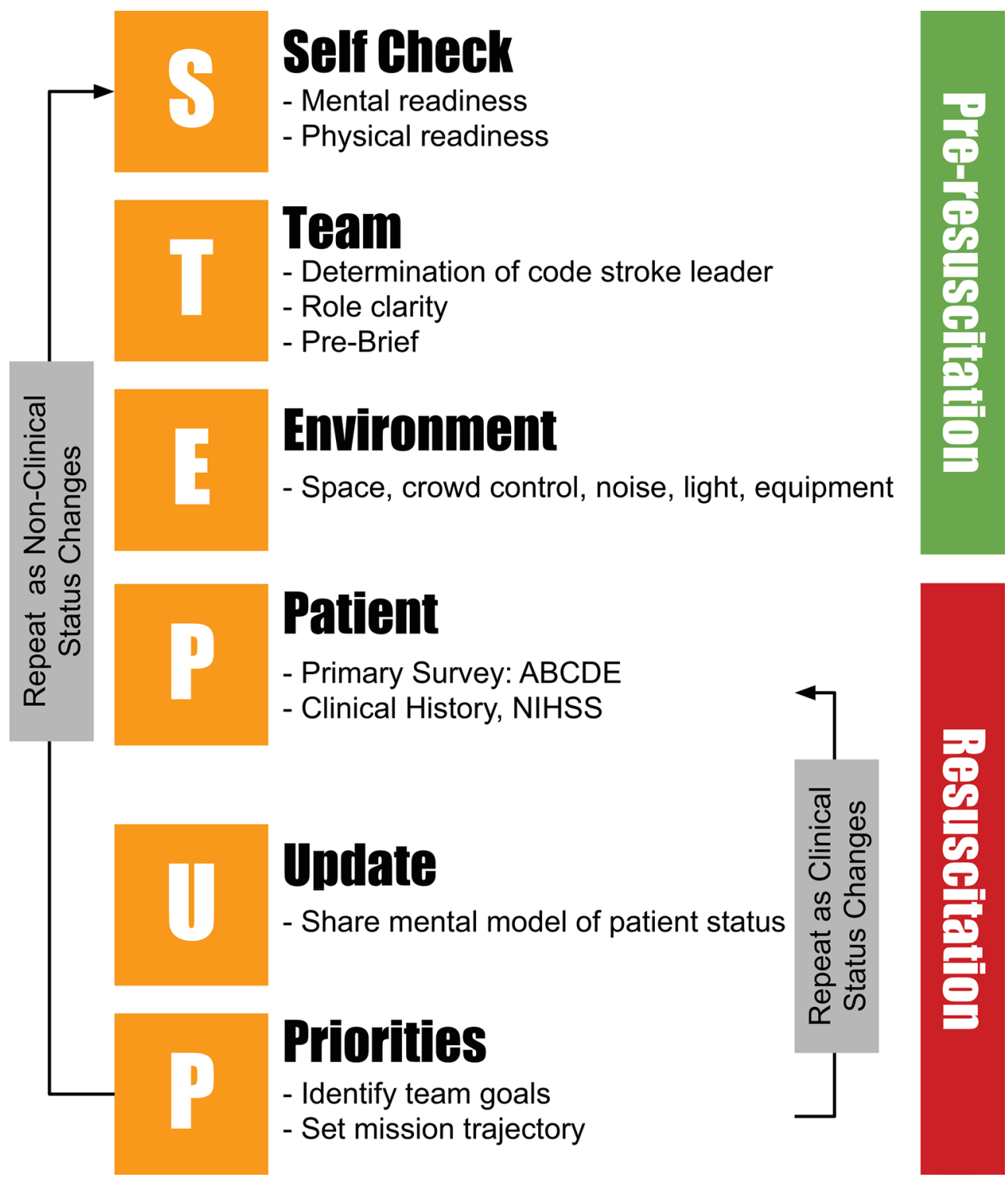

Fig. 1 Zero point survey. A framework for optimizing non-clinical processes before and during the code stroke (adapted from Reid et al.) [6]. Selfcheck refers to mental and physical readiness (stress and fatigue awareness, eating/elimination). While full mental readiness may not be possible at all times, small measures such as nourishment, purposeful attention to one's physiology (breathe, talk, see, focus) can help optimize personal performance, reduce stress, and achieve additive marginal gains [29]. With each code, using this framework can achieve incremental preparedness, especially during protected codes, where additional steps and personal protective equipment are required. Role clarity, attention to the environment/patient, sharing the mental model are each key ingredients of crisis resource management. ABCDE, airway, breathing, circulation, disability, exposure

services such as neuroangiography, medical imaging, and critical care) while triaging their effectiveness to achieve a desired outcome is imperative. Optimizing triage and prioritization strategies using knowledge gained from previous code stroke debriefings and simulation sessions can be effective in developing mitigation strategies to 
combat task saturation. The team leader often facilitates triage and prioritization; however, maintenance of situational awareness, while remaining flexible and sharing the mental model, can provide cognitive off-loading. In case of simultaneous code strokes, rapid triaging of each patient and tasks at hand while leveraging assistance from other teams is essential to providing stability during dynamic and stressful scenarios. Completion of a zero point survey prior to patient arrival can help reiterate priorities and facilitate team discussion around potential contingency plans if unexpected changes in patient condition occur.

\section{Cognitive Load}

As a high-stakes scenario, decision-making in stroke resuscitation presents itself as cognitively taxing. Cognitive load theory posits that human cognition has a limited working memory, capable of holding a finite amount of information simultaneously [8]. This limitation is exclusive to novel information acquired through sensory processes and is not impacted by information stored in long-term memory. Ability to interpret and analyze new information may be inhibited by extraneous information that would distract from the task at hand. This theory proposes that clinicians can develop expertise through repeated exposure to similar clinical events that develop into schemas that can be stored in long-term memory for future use. Novice clinicians with limited experience can decrease their cognitive load by off-loading any mentally taxing tasks to other team members and using memory aids and tools to decrease reliance on faulty memory $[9$, 10].

Cognitive load is increasingly recognized as a barrier to situational awareness and high-performing teams. Two models that are used to provide a framework for clinical decision-making are the Dual Process model [11] and Recognition-Primed Decision (RPD) Model [12]. The former construct divides thinking and decision-making into "System I" which is characterized by fast judgments that are based on intuition and experience. "System II" is more analytical. System I thinking is credited with allowing individuals to come to decisions quickly, based on learned responses from repeated exposure to previous similar presentations. System II is slower, lending itself to situations that require more deliberate, logical reasoning. Over time, experienced providers further develop System I from System II. Nonetheless, there is a need to re-frame and go back to System II thinking in times of stress when actions within a resuscitation are not having the desired effect. This is a key attribute of an experienced provider who has undergone stress inoculation and gained the cognitive skills to switch between modes as applicable.
Some interpretations of this model have attributed medical error to an overreliance on the reflexive nature of System I thinking [13] and a failure to take a deliberate analytical approach with System II thinking. This may be further complicated by implicit bias, where a learned set of schemas associated with a specific presentation may prevent the clinician from considering alternative diagnoses. A simplistic example in the hyperacute stroke population would be failing to check blood glucose level in the initial assessment and consider hypoglycemia as a potential stroke mimic. However, one can argue that both Systems are beneficial in their own respects, and diagnostic errors cannot be simply prevented with cognitive forcing strategies $[14,15]$.Clinicians must recognize that decision-making may be influenced by both Systems and be aware of their implicit bias.

During rapid task switching between examining the patient, obtaining the history, and other tasks, the experienced and collected provider will prioritize tasks, delegate to other team members, and re-evaluate the value-add of each step-in order to achieve a desired outcome. Unnecessary steps are removed to reduce cognitive clutter and time. When dealing with a suspected or confirmed COVID-19 patient, fear of exposure is a significant source of cognitive stress and anxiety for the provider. Presence of a "buddy system" as in the case of the safety lead, meticulous attention to donning/doffing procedures, and communication between the resuscitation leader and members outside of the room can off-load the cognitive burdens.

In an increasingly digital environment and need for physical distancing and ventilation, future design of resuscitation areas and digital tools used by providers need to include CRM principles such that distractions are kept to a minimum and important alerts and facts are able to come to the attention of the team in a prompt manner, all while maintaining a physical environment that provides space, adequate ventilation, but also a layout that eases cognitive load [16].

Despite best efforts to balance cognitive load, dynamics of a code stroke yield naturally to disruption impacting attention and increasing cognitive burden. This can be in the form of simultaneous patient arrivals and/or sudden deterioration, or events such as attention requested by a team member from either within or outside the resuscitation team. In such cases, acknowledgement of the individual and their request, an actionable plan with good communication is imperative. Strategies that can be utilized to manage disruption include task delegation, prioritization, but also fostering an environment where team members maintain a collective unified situational awareness focused on the mission at hand, while acknowledging the need to attend to the disruption. 


\section{Role Clarity}

Clear understanding of team members' roles and responsibilities optimizes team dynamics and maximizes efficiencies. It ensures that all clinical care is carried out efficiently, while minimizing duplication, and preventing errors of omission. A briefing prior to patient arrival can help with introduction to team members, ensuring members know each other by name, which is a common challenge with ad hoc teams. To ensure appropriate role designation, the code leader should ideally be familiar with the skills, capability, and experience of the other team members. Likewise, team members should be aware of each other's capabilities and limitations while adapting to dynamic situations.

Note that role designation can further be optimized by spatial allocation of team members during resuscitation. Implementation of a pit crew type approach with spatial considerations has previously been validated in cardiopulmonary resuscitation (Fig. 2) [17].

\section{Communication}

Communication is not only essential to the delivery of high-quality hyperacute stroke care, but is essential to the entire care pathway. Breakdown in communication is one of the leading causes of medical error and can lead to patient harm [18]. As such, it is another pillar of CRM, and efforts should be made to explicitly teach the principles of effective verbal communication to all members of the health-care team.

Ingredients of successful communication include closed loops of communication, which means that a piece of information is vocalized by a team member, repeated by the receiving clinician, and reported back. During a code stroke, entry of orders, communication with diagnostic imaging staff, and medication administration are such examples. If and when there is a breakdown in communication, it is the role of the code leader to briefly stop everyone from speaking, step back, and clarify roles and tasks moving forward.

In high-stakes situations, brevity of words and choosing them wisely is helpful. Stating key information in a calm, collected manner is essential. This is important during resuscitation but also during each handover of care points such as transfer to other units, centers, or operative settings [2]. Cognitive load is high and thus maintaining utmost precision and accuracy in communication is essential.

Although communication among team members is essential, communication with the patient and/or their family members should not be neglected. The code leader should assign a team member this responsibility, which is especially important when informed consent is required for hyperacute treatments like thrombectomy.

\section{Debriefing}

After the initial resuscitation period is complete, a debriefing should be held between all team members. Ideally, this is performed shortly after the acute scenario as a "hot debrief," proposed herein for a stroke-specific use case (Fig. 3). This is especially important in cases with suboptimal outcome or safety issues. Debriefing has military origins where soldiers would gather after a mission to review events to reduce psychological stress. Originally having an emphasis on error identification, the focus shifted over time to non-punitive guided group discussions and reflection [19]. It also has deep roots in the aviation industry, where many of the CRM principles are based. In the clinical environment, debriefing has been utilized after critical events to discuss individual performance, knowledge, and cognitive decision-making [20]. It has been found to develop CRM skills in health profession students [21], decrease mental workload [22], and potentially improve clinical outcomes [23].

Admittedly, debriefing in the clinical environment is not without its challenges. Competing patient care demands [24], lack of an appropriate space in the clinical area, disinterest by some team members, insufficient support from administration, and fear of being scrutinized for failures [25] can all pose barriers to successful implementation. Debriefing facilitators must create a psychologically safe environment to promote dialogue between all team members [20]. Given the unique challenges of hosting immediate debriefing in the clinical environment, some authors have provided tangible strategies for successful implementation $[20,26]$.

\section{Simulation}

While conceptually simple, translation of these nontechnical skills into clinical practice requires deliberate and repeated exercise. Simulation provides an opportunity for clinicians to have repeated exposure to variations in a clinical scenario in a safe environment. With repeated exposure to simulated high-stakes situations, clinicians undergo stress inoculation and will be more likely to recognize immediate threats and respond appropriately in a controlled manner. In one systematic review, it was shown that CRM skills learned during simulation training were effectively transferred to the clinical setting with an improvement in patient outcomes and even mortality [27]. Furthermore, simulation is able to address some of the communication challenges encountered with ad hoc teams. In one study, participants changed their communication behaviors and teamwork following brief training exercises [28]. However, there was some loss of retention 


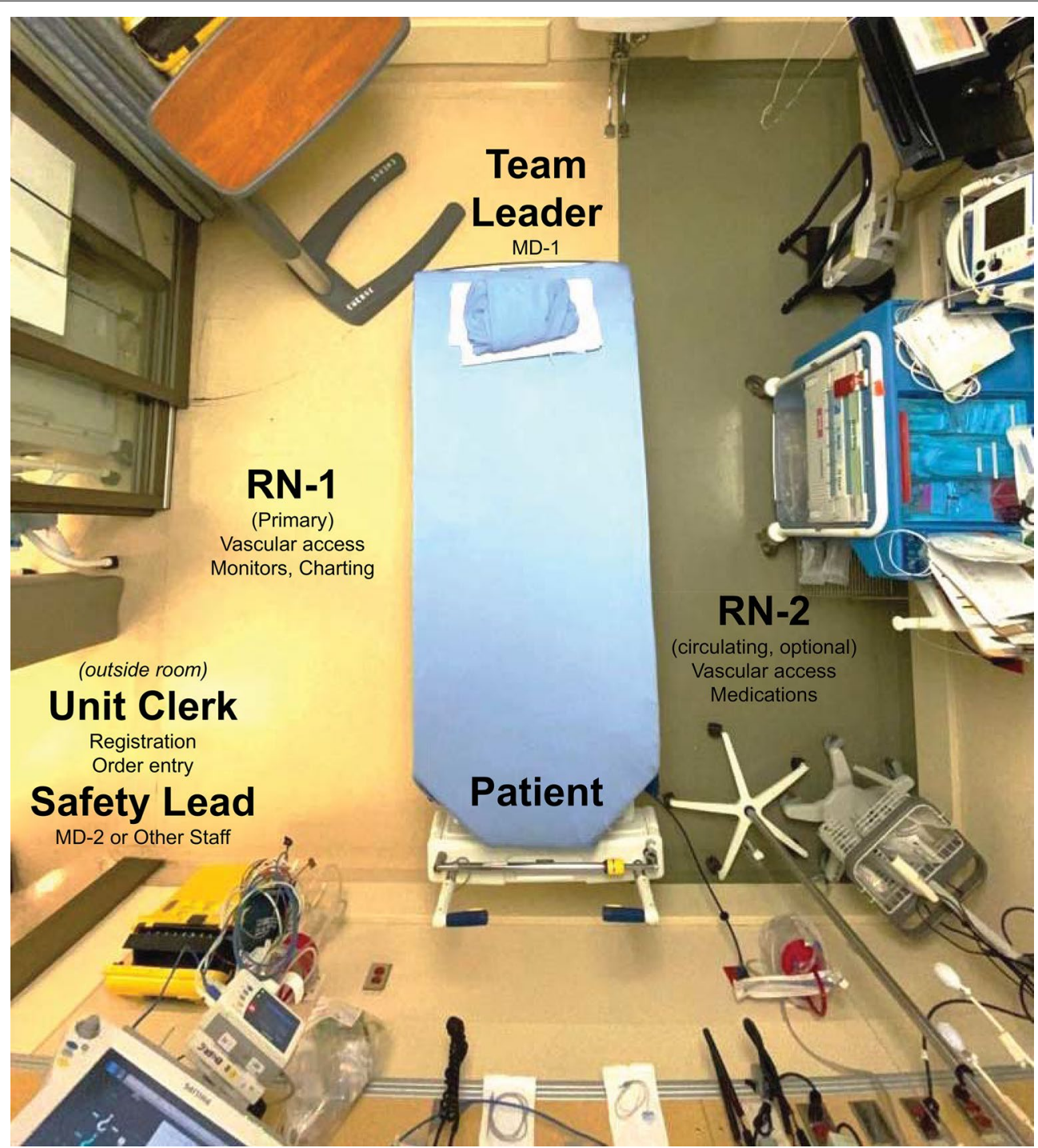

Fig. 2 Spatial allocation facilitating team role designation. Suggested spatial allocation of the team during a code stroke resuscitation. This should be modified as needed for local practice parameters. MD-1 is the team leader responsible for initiating the zero point survey pre-resuscitation, performing the primary survey and NIHSS assessment, and facilitating the stroke checklist prior to departure to imaging. In the context of a protected code stroke, the in-room team is optimized to two individuals MD-1, RN-1. RN-2 is optional depending on the clinical need as are other team members such as a second MD (e.g., for airway management), and respiratory therapist (RT) as applicable. The key designation of a safety lead who is outside the room ensuring proper donning and doffing of PPE during protected code stroke procedures remains important. In isolation/protected scenarios, use of a handsfree speaker and microphone (in-room portable monitors) for two-way communication is effective. Similarly, single-use stickers (e.g., designated as "code leader," RN (primary),"'Safety Officer") are worn by members on their clothing or PPE gowns as applicable

over time, emphasizing the need for ongoing regular training and simulation. We use in situ simulation, with ad hoc teams, and utilize a checklist for each code stroke to foster CRM principles (Fig. 4).

\section{Concluding Remarks}

CRM principles are adopted and well established in resuscitative disciplines including in specialized neurocritical care environments. There may be barriers to widespread adoption in general critical care settings and more so in all emergency health-care settings. These include availability of personnel, resources, education, and local practice priorities. However, this pandemic provides a unique opportunity to consider implementation of CRM for emergency preparedness and especially in acute stroke care. A consistent approach within an organization, while respecting local practice parameters, ensures a system of readiness, 


\section{Hot Debriof}

\section{Initiate}

- Announce to the team that a quick debriefing will occur

- Encourage all team members to participate

\section{Safe Space}

- Ensure psychological safety and a flat hierarchy

- Create a non-threatening, no-blame environment

\section{Review}

- Discuss chronological progression of events

- Allow team members to provide their own perspectives

\section{Team Successes}

Team Challenges

- Discuss personnel, process, and equipment issues

- Discuss team communication and barriers

- Avoid critique of individual performance

- Avoid jumping to a solution

\section{Participate}

- Ensure all team members have an equal opportunity to speak

\section{Follow-up}

\section{- Track and follow-up on voiced concerns}

- Allow option for individuals to discuss concerns privately

Fig. 3 Hot Debrief. Code stroke debrief is performed optimally shortly after the conclusion of resuscitation, away from the patient clinical area, and after the patient has been treated and stabilized. All available team members should be encouraged to participate. The debrief promotes a flat hierarchy where all members can equally contribute, and voice concerns of items requiring improvement and follow-up. In addition, team members provide positive feedback about elements that facilitated an effective resuscitation. Debriefing facilitators create a psychologically safe environment to promote dialogue between the team. Each of the steps above utilizes principles of crisis resource management

evaluation, and identification of areas for ongoing improvement and harmonization across programs. Both hyperacute stroke care and follow-up care provided on stroke units rely heavily on multidisciplinary teams. Systems and pathways must develop the ability to adapt rapidly to acute and emergent stressors such as the ongoing COVID-19 pandemic. The therapeutic tools we have in stroke are only as good as the teams working together to deliver them to the patient. CRM is uniquely poised to address the current need and future emergency preparedness in stroke care. In order to be prepared for future adversities with high-performing 


\section{Strotre HieGhist PRIOR to Pationt Aritual}

Activate Code Stroke via Emergency Line

Initiate pre-resuscitation Zero Point Survey

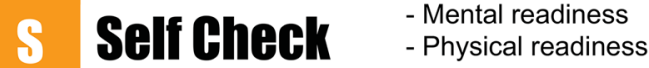

- Determination of code stroke leader

TAIII - Role clarity, Safety Lead designation

- Pre-Brief

Environment - Space, crowd control, noise, light, equipment

1) Patipnt - Primary Survey: ABCDE

Patient - Clinical History, NIHSS

\section{PRIOR to Denarture fiom issessment Room}

$\checkmark$ Are we satisfied with the primary survey?

$\checkmark$ Is the transport monitor \& thrombolysis kit ready?

$\checkmark$ IV access established and functioning?

$\checkmark$ Has bloodwork been sent?

\section{Is GT ready?}

\section{Do we need additional equipment or medications?}

Fig. 4 Stroke checklist. A checklist is used for in situ stimulation and performed prior to patient arrival with the pre-resuscitation portion of the zero point survey. This should be modified and adapted as needed to conform with local practice parameters. This checklist has a second portion performed prior to departure for acute imaging in order to ensure that all elements are performed for expedited progress into downstream processes

teams, routine implementation of CRM will be of added value.

\section{Author details}

${ }^{1}$ Brain Resuscitation Lab, Neurology Quality and Innovation Laboratory (NQIL), Division of Neurology, Department of Medicine, Sunnybrook Health Sciences Centre, University of Toronto, Room H335 - 2075 Bayview Avenue, Toronto, ON M4N 3M5, Canada. ${ }^{2}$ Department of Emergency Services, Sunnybrook Health Sciences Centre, Lawrence S. Bloomberg Faculty of Nursing, University of Toronto, Toronto, ON, Canada. ${ }^{3}$ Department of Emergency Medicine, Northern Beaches Hospital, Frenchs Forest, Sydney, Australia. ${ }^{4}$ Department of Emergency Medicine and Acute Stroke Service, Yale School of Medicine, New Haven, USA. ${ }^{5}$ Division of Neurosciences Critical Care, Departments of Anesthesiology and Critical Care Medicine, Neurology, and Neurosurgery,
The Johns Hopkins University School of Medicine, Baltimore, USA. ${ }^{6}$ Division of Emergency Critical Care, Stony Brook Medicine, Stony Brook, USA.

\section{Acknowledgements}

We would like to thank Dr William J. Powers (Department of Neurology, University of North Carolina School of Medicine) for his review of the manuscript and providing comments and insights that were used to revise it. We acknowledge members of our door-to-needle quality improvement project group who have been involved in facilitating a modified version of the stroke checklist: Elizabeth Linkewich, Sandy Lyeo, Catherine Convery, and Will Thomas-Boaz.

\section{Authors' Contribution}

This manuscript complies with all instructions to authors. All authorship requirements have been met, and the final manuscript was approved by all authors. 


\section{Conflict of interest}

The authors declare that they have no conflicts of interest.

\section{Ethical Statement}

This study adheres to ethical guidelines. An IRB was not required for this study.

\section{Publisher's Note}

Springer Nature remains neutral with regard to jurisdictional claims in published maps and institutional affiliations.

\section{Received: 18 May 2020 Accepted: 20 July 2020}

Published online: 13 August 2020

\section{References}

1. Khosravani H, Rajendram P, Notario L, Chapman MG, Menon BK. Protected code stroke: hyperacute stroke management during the Coronavirus Disease 2019 (COVID-19) Pandemic. Stroke 2020:STROKEAHA120029838.

2. Optimizing crisis resource management to improve patient safety and team performance: a handbook for all acute care health professionals. Royal College of Physicians and Surgeons of Canada, 2017. http://www. royalcollege.ca/rcsite/ppi/educational-resources-e. Accessed 20 April 2020.

3. Bleetman A, Sanusi S, Dale T, Brace S. Human factors and error prevention in emergency medicine. Emerg Med J. 2012;29:389-93.

4. Haerkens MH, Jenkins DH, van der Hoeven JG. Crew resource management in the ICU: the need for culture change. Ann Intensive Care. 2012:2:39.

5. Gaba DM. Crisis resource management and teamwork training in anaesthesia. Br J Anaesth. 2010;105:3-6.

6. Reid C, Brindley P, Hicks C, et al. Zero point survey: a multidisciplinary idea to STEP UP resuscitation effectiveness. Clin Exp Emerg Med. 2018;5:139-43.

7. There is no emergency in a pandemic. 2020. https://acanticleforlazarus. com/2020/03/23/there-is-no-emergency-in-a-pandemic/. Accessed July 7, 2020

8. van Merrienboer JJ, Sweller J. Cognitive load theory in health professional education: design principles and strategies. Med Educ. 2010;44:85-93.

9. Luten R, Wears RL, Broselow J, Croskerry P, Joseph MM, Frush K. Managing the unique size-related issues of pediatric resuscitation: reducing cognitive load with resuscitation aids. Acad Emerg Med. 2002;9:840-7.

10. Fletcher JL. AANA Journal course: update for nurse anesthetists-ERR WATCH: anesthesia crisis resource management from the nurse anesthetist's perspective. AANA J. 1998;66:595-602.

11. Kahneman D. Maps of bounded rationality: psychology for behavioral economics. Am Econ Rev. 2003;93:1449-75.

12. Klein GA. A recognition-primed decision (RPD) model of rapid decision making. In: Klein GA, Orasanu J, Calderwood R, Zsambok CE, editors. Decision making in action: models and methods. Norwood: Ablex Publishing Corporation; 1993. p. 138-47.
13. Croskerry P. A universal model of diagnostic reasoning. Acad Med. 2009;84:1022-8.

14. Monteiro SD, Sherbino J, Patel A, Mazzetti I, Norman GR, Howey E. Reflecting on diagnostic errors: taking a second look is not enough. J Gen Intern Med. 2015:30:1270-4.

15. Quinn A, Robinson D, Tobias A. Chapter 9: dual-process reasoning. In: Chan TM, Gottlieb M, Sherbino J, Boysen-Osborn M, Papanagnou D, Yarris L, editors. Education theory made practical. San Franciso: Academic Life in Emergency Medicine; 2017.

16. Cognitive Load and Its Implications for Health Care. NEJM Catalyst, 2018. https://catalyst.nejm.org/doi/full/10.1056/CAT.18.0233. Accessed 7 July 2020

17. Hopkins CL, Burk C, Moser S, Meersman J, Baldwin C, Youngquist ST. Implementation of pit crew approach and cardiopulmonary resuscitation metrics for out-of-hospital cardiac arrest improves patient survival and neurological outcome. J Am Heart Assoc. 2016;5:1.

18. Woolf SH, Kuzel AJ, Dovey SM, Phillips RL Jr. A string of mistakes: the importance of cascade analysis in describing, counting, and preventing medical errors. Ann Fam Med. 2004;2:317-26.

19. Hunter LA. Debriefing and feedback in the current healthcare environment. J Perinat Neonatal Nurs. 2016;30:174-8.

20. Berg GM, Hervey AM, Basham-Saif A, Parsons D, Acuna DL, Lippoldt D. Acceptability and implementation of debriefings after trauma resuscitation. J Trauma Nurs. 2014;21:201-8.

21. Coppens I, Verhaeghe S, Van Hecke A, Beeckman D. The effectiveness of crisis resource management and team debriefing in resuscitation education of nursing students: a randomised controlled trial. J Clin Nurs. 2018;27:77-85

22. Boet S, Sharma B, Pigford AA, Hladkowicz E, Rittenhouse N, Grantcharov T. Debriefing decreases mental workload in surgical crisis: a randomized controlled trial. Surgery. 2017:161:1215-20.

23. Couper K, Salman B, Soar J, Finn J, Perkins GD. Debriefing to improve outcomes from critical illness: a systematic review and meta-analysis. Intensive Care Med. 2013;39:1513-23.

24. Nadir NA, Bentley S, Papanagnou D, Bajaj K, Rinnert S, Sinert R. Characteristics of real-time, non-critical incident debriefing practices in the emergency department. West J Emerg Med. 2017;18:146-51.

25. Mullan PC, Kessler DO, Cheng A. Educational opportunities with postevent debriefing. JAMA. 2014;312:2333-4.

26. Kessler DO, Cheng A, Mullan PC. Debriefing in the emergency department after clinical events: a practical guide. Ann Emerg Med. 2015:65:690-8

27. Boet $S$, Bould MD, Fung $L$, et al. Transfer of learning and patient outcome in simulated crisis resource management: a systematic review. Can J Anaesth. 2014;61:571-82.

28. Roberts NK, Williams RG, Schwind CJ, et al. The impact of brief team communication, leadership and team behavior training on ad hoc team performance in trauma care settings. Am J Surg. 2014;207:170-8.

29. Lauria MJ, Gallo IA, Rush S, Brooks J, Spiegel R, Weingart SD. Psychological skills to improve emergency care providers' performance under stress. Ann Emerg Med. 2017:70:884-90. 